\title{
Editorial
}

\section{THE SAGA OF THE SKIN SMEAR}

Three methods of bacteriological investigation of leprosy patients were in vogue by the time the "scraped incision" method became generally accepted as the most practical. There was the very first one, described by L. J. Alvarez at the 1st International Leprosy Congress, held in Berlin in 1897; this was to remove a small piece of skin surgically, grind it up in a mortar with a few drops of saline, and make smears of the resultant fluid. The second was the "skinclip" method, favoured by the late Ernest Muir (1921), which involved clamping the surface of a skin lesion with curved haemostatic forceps and snipping off a small piece of skin with curved scissors; the raw surface of the clipping was then rubbed or squeezed onto a slide. The third was the puncture method which the late $\mathrm{H}$. W. Wade found in routine use in Manila when he was appointed to the official Leper Examining Committee of the Bureau of Health in 1916. Here are his own words (Wade, 1963):

"The main function of the Committee was the examination of smears for bacilli. The expression often used in those days was 'examinar el sangre', and it was truly blood that was examined. Before the Committee convened, the resident staff had prepared the smears. Usually selecting a single lesion of each patient, it was punctured deeply with a surgical needle. The blood that exuded, or was expressed, was smeared more or less widely and thickly on a slide. When lesions contained an abundance of bacilli there was no difficulty in the examination, but it was otherwise when they contained few or none. In such cases it was required that each member of the Committee should, in turn, spend a certain number of minutes on each slide before a patient could be declared negative and eligible for release. That was very time-consuming, and exquisitely boresome."

In the following year (1917) Wade replaced this puncture method by his "scraped incision" method, but it was not until ten years later that it was published in a booklet written with Rodriguez (1927), and it was not contributed to the periodical literature until nearly another decade had elapsed; this was a revised description of the method by Wade (1935), so detailed and clear that it could be copied verbatim into any modern leprosy textbook. At that time it was usual for smears to be taken exclusively from skin lesions, as is modern practice in non-lepromatous leprosy, and time had to pass before earlobes were included in routine smears in lepromatous leprosy, and for many years it has been generally accepted that bacilli are likely to be found in earlobes, in long-treated patients, when they have disappeared from other sites. The wheel of progress has made a further turn, and the earlobe has now been 
replaced by the finger as the richest site for bacilli in such patients, and also the skin site from which the earliest intimation of relapse can be obtained by finding solid-staining bacilli whereas such (living) bacilli had been absent previously. It was at the Hospital for Tropical Diseases in London that the original work was done on the inclusion of skin smears from fingers in followup tests on lepromatous patients under treatment (Ridley et al., 1976). The idea came from Mrs Marian Ridley and was prompted by a conversation she had with Dr John Pearson in which the latter queried why fingers should so often appear oedematous even when there are no clinical lesions there. After discussing the programme with her I began to include two finger smears in all lepromatous patients reporting for follow-up examination, and I decided to use the dorsum of each middle finger. The first phalanx was chosen as it seemed easier to make a good smear because there was enough skin to grip between thumb and index finger of the left hand. The following smears were taken from each of 30 patients reporting consecutively in 1975: 2 from earlobes, 2 from healing or healed lesions on trunk or limbs, and 2 from fingers. Additional smears were from toes and nasal mucosa in some patients. Our results showed that the mean Bacterial Index (BI) was a little higher, and the mean SFG Index was much higher, in fingers. In four patients the fingers were the sole skin site for bacilli. Toes gave results which were less informative than fingers, and as would be expected in long-treated patients, nasal mucosa was generally unrewarding, but the nose gave early intimation of impending relapse in one patient as the few bacilli seen were all solid-staining; finger smears gave supporting evidence in this case as some solid-staining bacilli were present among the fragmented and granular bacilli. No solids were found at other sites.

The next report (Hiramalini et al., 1978) was on 41 lepromatous patients receiving treatment at Karigiri, India. Smears were taken from both middle fingers, both second toes, right earlobe, left forehead, right chin, left buttock, and nasal mucosa. All patients wore sandals. The authors found fingers and toes consistently more informative than the other sites, with higher BI and MI, but with no significant difference between them, excepting that the terminal phalanx of the finger harboured more solid-staining bacilli than the middle phalanx. Three patients with negative smears from earlobes and nose had granular bacilli in fingers and toes, and in one patient who was negative at all routine sites after 10 years of treatment, granular bacilli were found only in the terminal phalanges of fingers and toes.

The paper by Haidar Abu Ahmed et al. in the present Number of this Journal compares smears from eight different skin sites in 18 new (untreated lepromatous patients. They found that mean values of BI were highest in earlobes and fingers $(4.5+$ in each), and as regards MI the figure for earlobes was slightly higher than that for fingers (14.3 as against 13.0). We are not told which phalanx was chosen. The authors recommend 6 smears from all new lepromatous patients and during follow-up tests: 2 from earlobes, 1 from face, 1 from buttock, and 2 from fingers.

In another paper in this Number, Jopling et al. take the story of finger smears a stage further. They describe two patients, under treatment with dapsone, and showing no clinical signs of relapse, who were found on routine testing to have solid-staining bacilli in fingers. Mouse foot-pad inoculation gave 
positive results in both cases (proving that viable bacilli were present in the fingers), and in the second patient tests for dapsone resistance were carried out; these confirmed the presence of dapsone-resistant organisms.

These four papers leave no doubt that smears from fingers should be included in the assessment of all new lepromatous patients and in all follow-up examinations, and I would go so far as to suggest that no more than 4 smears are necessary: one from each earlobe and one from the terminal phalanx of each middle finger.

At this stage in our knowledge we can do no more than surmise as to the reasons why in lepromatous leprosy the skin of fingers is so rich in bacilli, and there are three likely explanations, working in concert: firstly, fingers are among the coolest skin sites, and it is well known that leprosy bacilli prefer cooler sites; secondly, the dermis of fingers has a good supply of sensory nerves, much favoured by bacilli; thirdly, these dermal nerves on the dorsal aspects of fingers are close to the surface because of the thinness of the dermis, and therefore an incision with a scalpel blade can readily collect bacilli from them; this applies particularly to the middle and distal phalanges. The role of repeated minor trauma is more speculative.

W. H. JOPLING

\section{References}

Hiramalini, S., Joseph, N. A. and Chacko, C. J. G. (1978). Concentration and persistence of bacilli in the fingers and toes of patients with lepromatous leprosy. Lepr. Rev. 49, 223.

Muir, E. (1921). Handbook on leprosy: its diagnosis, treatment and prevention. Cuttack: R. J. Grundy.

Ridley, M. J., Jopling, W. H. and Ridley, D. S. (1976). Acid-fast bacilli in the fingers of longtreated lepromatous patients. Lepr. Rev.47,93.

Wade, H. W. and Rodriguez, J. N. (1927). A description of leprosy: its etiology, pathology, diagnosis and treatment. Manila: Bureau of Printing.

Wade, H. W. (1935). The bacteriological examination in leprosy. Lepr. Rev. 6, 54.

Wade, H. W. (1963). The examination of skin lesions for bacilli. Int.J. Lepr. 31, 242.

\section{Subscriptions to Leprosy Review, 1980 onwards}

At a meeting of the Editorial Board of Leprosy Review in August 1979, it was decided that the yearly subscription, from Number $1,51,(1980)$ would be $£ 15$ for the four issues; or $£ 4$ per copy, inclusive of postage and packing (UK and abroad). Subscription orders or enquiries should be sent to the British Leprosy Relief Association (LEPRA), Fairfax House, Causton Road, Colchester, CO 1 1PU, England.At its own discretion, LEPRA will continue, and also expand, its policy of sending free issues of this journal to people in various parts of the world; this will include doctors working directly with leprosy who cannot afford the above subscription, or obtain foreign currency, together with selected libraries covering tropical medicine. 